\title{
Determination of thickness and dielectric constant of thin transparent dielectric layers using Surface Plasmon Resonance
}

\author{
Helene E. de Bruijn, Bert S.F. Altenburg, Rob P.H. Kooyman and Jan Greve \\ Biophysical Technology Group, Faculty of Applied Physics, University of Twente, \\ P.O. Box 217, 7500 AE Enschede, The Netherlands
}

Received 26 December 1990

\begin{abstract}
The determination of the thickness and dielectric constant of thin dielectric layers by means of surface plasmon resonance is discussed. It appears to be impossible to determine these parameters from one surface plasmon response experiment. This is illustrated theoretically. Variation of the refractive index of the solution in which surface plasmon experiments were performed allowed us to determine these parameters separately.
\end{abstract}

\section{Introduction}

Surface Plasmon Resonance (SPR) occurs when light is reflected at a specific angle $\theta_{\mathrm{p}}$, the plasmon angle, in a glass substrate onto which a thin metal layer has been evaporated. SPR is detected by the decrease in reflection coefficient which occurs at incidence angles close to $\theta_{\mathrm{p}}$ and by the minimum at $\theta_{\mathbf{p}}$ itself. The plasmon angle depends strongly on the thickness and dielectric constant of a dielectric layer present on top of the silver layer. Therefore SPR has become very important for applications in chemooptical sensors [1,2], which are designed to detect variations in thickness or dielectric constant of thin dielectric layers.

A SPR reflection curve can be described by three parameters: the position of the minimum, the minimum reflection coefficient and the half-width. If no dielectric layer is present on top of the metal layer, two solutions for the thickness and complex dielectric constant of the metal layer are obtained from these three parameters [3]. After performing one more experiment or by using additional information the correct solution can be determined.

In the study of the properties of solid/liquid interfaces, at which monomolecular layers can be adsorbed, it would be very useful if the average thickness and dielectric constant of such a layer could be separately determined. Knowledge of these quan- tities provides more detailed information on the nature of the adsorbed state.

Initially it was anticipated that a dielectric layer on top of the metal layer could be characterized in a similar way by its thickness and dielectric constant. It appeared, however, impossible to determine the thickness and dielectric constant of a thin nonabsorbing layer separately. In this paper we discuss some alternative experimental procedures which make this determination possible. Experimental results of one such procedure will be presented, together with a discussion of the accuracy that can be obtained.

\section{Theory}

The layer system we want to describe is shown in fig. 1. The actual problem is how to calculate the average thickness $d_{3}$ and dielectric constant $\epsilon_{3}$ from an experimental SPR curve. We define the characteristic parameters for the SPR curve in fig. 2, where the reflection coefficient $R$ is depicted as a function of the wavevector component $k_{x}$ parallel to the interfaces.

The value of the reflection coefficient of the layer system follows from the Fresnel equations for p-polarized light, which can be written as 


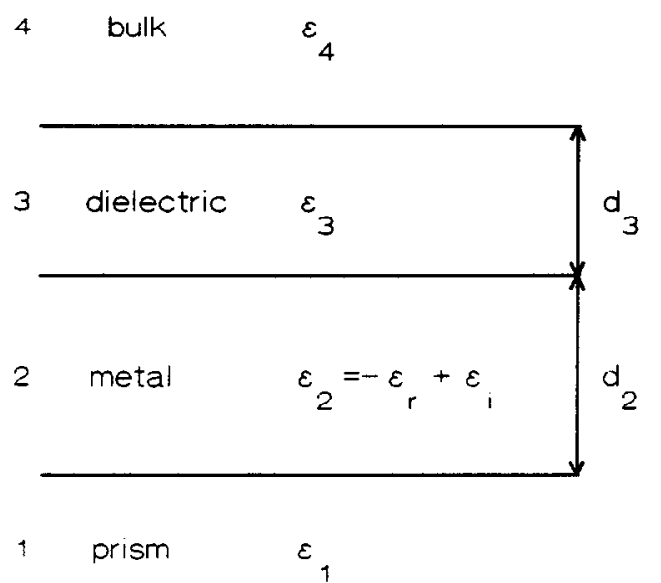

Fig. 1. Definition of the layer system.

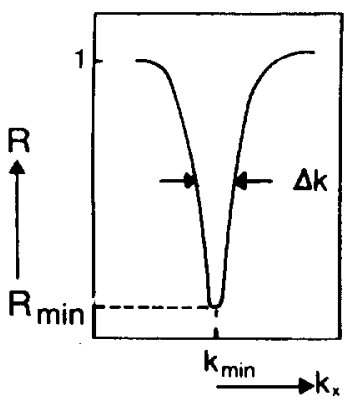

Fig. 2. Definition of the characteristics of the SPR reflection curve.

$r_{1234}=\frac{n_{234}+z_{234} r_{12}^{-1} \exp \left(2 \mathrm{i} k_{z 2} d_{2}\right)}{n_{234}+z_{234} r_{12} \exp \left(2 \mathrm{i} k_{z 2} d_{2}\right)} r_{12}$,

with

$r_{234}=\frac{z_{23} n_{34}+z_{34} n_{23} \exp \left(2 i k_{z 3} d_{3}\right)}{n_{23} n_{34}+z_{23} z_{34} \exp \left(2 i k_{z 3} d_{3}\right)} \equiv \frac{z_{234}}{n_{234}}$,

$r_{i j}=\frac{\epsilon_{j} k_{z i}-\epsilon_{i} k_{z j}}{\epsilon_{j} k_{z i}+\epsilon_{i} k_{z j}} \equiv \frac{z_{i j}}{n_{i j}}$

$k_{z i}$ is the wavevector component perpendicular to the interface in medium $i$. It is impossible to obtain an exact analytical expression for $k_{\min }, \Delta k_{1 / 2}$ and $R_{\min }$ as a function of $d_{3}$ and $\epsilon_{3}$. To circumvent this problem we approximate eq. (1); first we define $k_{x}=k_{x}^{0}+\Delta k_{x}$, where $k_{x}^{0}$ is a good approximation of the plasmon wave vector if no dielectric layer is present $[3,4]$, $k_{x}^{0}=(\omega / c) \sqrt{\epsilon_{4} \epsilon_{r} /\left(\epsilon_{\mathrm{r}}-\epsilon_{4}\right)}$.

Now we make the following approximations:

- since we are only interested in thin dielectric layers, $k_{\min }$ will not be very different from $k_{x}^{0}$ and we can write

$$
\begin{aligned}
k_{z j} & =\sqrt{\epsilon_{j}(\omega / c)^{2}-k_{x}^{2}} \\
& \approx \sqrt{\epsilon_{j}(\omega / c)^{2}-k_{x}^{02}}-\frac{k_{x}^{0} \Delta k_{x}}{\sqrt{\epsilon_{j}(\omega / c)^{2}-k_{x}^{02}}} .
\end{aligned}
$$

- As the imaginary part of $\epsilon_{2}$ is small compared to the other dielectric constants and $\Delta k_{x}$ is small compared to the wavevector components, the terms $\epsilon_{i} \Delta k_{x}$ are neglected.

- From the fact that $d_{3}$ is small, we derive that we may write $\exp \left(2 \mathrm{i} k_{z 3} d_{3}\right) \approx 1-2 \mathrm{i} d_{3} k_{z 3}$ and we can neglect $\Delta k_{x}$ in $k_{z 3}$.

$-z_{234}$ is so large (order of $k_{x}^{0}$ ) that the contribution of $\Delta k_{x}$ to $z_{234}$ can be neglected.

Using these approximations the Fresnel reflection coefficient $r_{1234}$ from eq. (1) can be written in the form derived in appendix $\mathrm{A}$ and $k_{\min }$ can be determined. For the shift in $k_{\min }$ due to the presence of layer 3 one finds

$\Delta k_{\min } \approx d_{3}\left(\frac{2 \pi}{\lambda}\right)^{2} \frac{\left(\epsilon_{\mathrm{r}} \epsilon_{4}\right)^{3 / 2}\left(\epsilon_{3} \epsilon_{\mathrm{r}}-\epsilon_{3} \epsilon_{4}-\epsilon_{4} \epsilon_{\mathrm{r}}+\epsilon_{3}^{2}\right)}{\epsilon_{3}\left(\epsilon_{\mathrm{r}}^{2}-\epsilon_{4}^{2}\right)\left(\epsilon_{\mathrm{r}}-\epsilon_{4}\right)}$.

The difference between this approximated $\Delta k_{\min }$ and the exact $\Delta k_{\min }$ for a protein layer is smaller than $0.5 \%$. The change in half-width appears to depend in a similar way on $d_{3}$ and $\epsilon_{3}$, implying that no extra information can be obtained from this change. The reflection minimum $\boldsymbol{R}_{\min }$ does not change for thin transparent dielectric layers.

Eq. (3) gives a set of possible solutions for combinations of $\epsilon_{3}$ and $d_{3}$. For a silver layer with $d_{2}=$ $53 \mathrm{~nm}, \epsilon_{2}=-16.2+\mathrm{i} 0.52, \lambda=632.8 \mathrm{~nm}, \epsilon_{4}=1.78$ (water) and $\Delta k_{\min }=4.09 \times 10^{4} \mathrm{~m}^{-1}$ these solutions are shown in fig. 3 . That it is not due to the approximations that $d_{3}$ and $\epsilon_{3}$ cannot be determined separately is illustrated by the fact that the exact Fresnel equations yield only one SPR curve if four different $\epsilon_{3}, d_{3}$ pairs are chosen from the curve in fig. 3. One therefore needs additional independent experimental data to do a separate determination of $\epsilon_{3}$ 


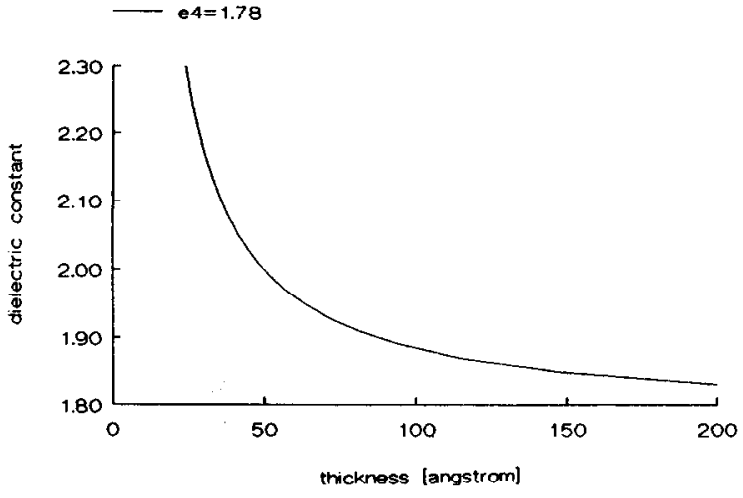

Fig. 3. Possible $\epsilon_{3}, d_{3}$ values of a dielectric layer on top of a silver layer with $d_{2}=53 \mathrm{~nm}, \epsilon_{2}=-16.2+0.52 \mathrm{i}, \lambda=632.8 \mathrm{~nm}, \epsilon_{4}=1.78$ (water) and $\Delta k_{\min }=4.09 \times 10^{4} \mathrm{~m}^{-1}$.

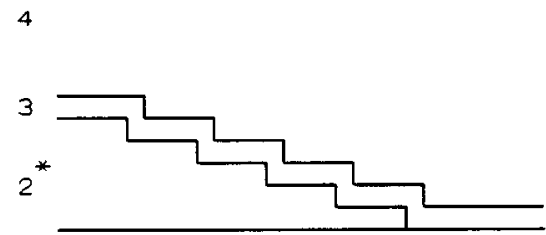

2

Fig. 4. Layer structure with additional layer $2^{*}$ with varying thickness to vary $\epsilon_{r}$.

and $d_{3}$. Apart from $\epsilon_{3}$ and $d_{3}$ the change in $k_{\min }$ depends on $\lambda, \epsilon_{\mathrm{r}}$ and $\epsilon_{4}$ [eq. (3)]. One can therefore vary one of these parameters to obtain more than one line of solutions for $d_{3}, \epsilon_{3}$. If these lines intersect, the true values of $\epsilon_{3}$ and $d_{3}$ follow from the intersection point.

The following comments can be made:

- Variation of $\lambda$ : A drawback is that $\epsilon_{\mathrm{r}}$ and $\epsilon_{3}$ will change as well and that $d \epsilon_{3} / \mathrm{d} \lambda$ should be known to apply this method.

- Variation of $\epsilon_{\mathrm{r}}$ : This can be done by varying $\lambda$, by using different metals; or by depositing a dielectric layer $2^{*}$ with varying thickness on top of the metal layer, as shown in fig. 4 . If we represent layers 2 and $2^{*}$ as one effective layer with thickness $d_{\text {eff }}$ and dielectric constant $\epsilon_{\mathrm{eff}}$, the real part of $\epsilon_{\mathrm{eff}}$ changes with the thickness of layer $2^{*} . d_{\mathrm{eff}}$ and $\epsilon_{\mathrm{eff}}$ can be deter-

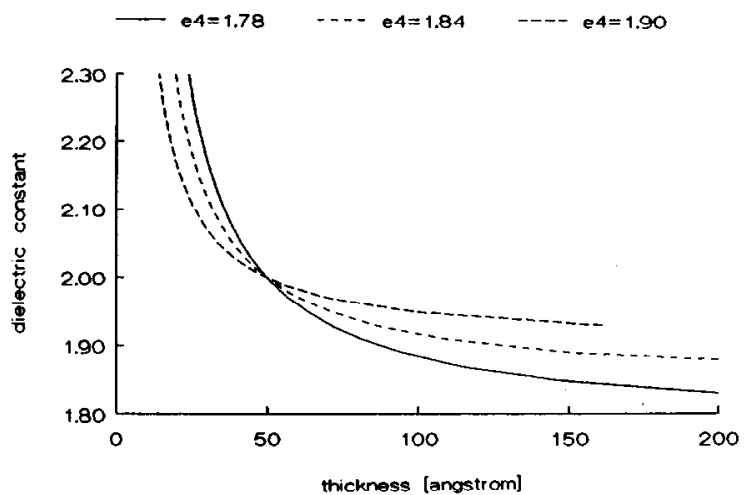

Fig. 5. $\epsilon_{3}, d_{3}$ solutions for $\epsilon_{4}=1.78,1.84$ and 1.90 in the same situation as fig. 3 .

mined from a SPR curve in a similar way as $d_{2}$ and $\epsilon_{2}$ of the metal layer are determined, which is described in ref. [1].

- Variation of $\epsilon_{4}$ : Eq. (3) can be rewritten in the form

$\Delta k_{\min }=\left(\frac{2 \pi}{\lambda}\right)^{2} \frac{\left(\epsilon_{\mathrm{r}} \epsilon_{4}\right)^{3 / 2}}{\left(\epsilon_{\mathrm{r}}-\epsilon_{4}\right)^{2}} \frac{d_{3}}{\epsilon_{3}}\left(\epsilon_{3}-\epsilon_{4}\right)$.

This shows that varying $\epsilon_{4}$ is a very effective method for determining $d_{3}$ and $\epsilon_{3}$ separately. As an example the calculated results for three values of $\epsilon_{4}$ are shown in fig. 5. It is clear that we obtain an intersection point from which the actual $d_{3}, \epsilon_{3}$ value can easily be determined.

\section{Materials and methods}

For the experiments we used the setup shown in fig. 6 . The laser is a polarized $10 \mathrm{~mW} \mathrm{HeNe} \mathrm{laser}$

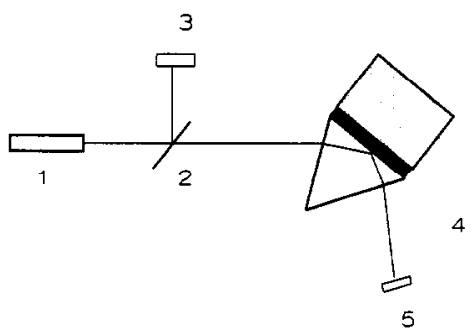

Fig. 6. Experimental setup. The intensity of the HeNe laser (1) is measured with a beamsplitter (2) and a reference photodiode (3). The prism-cuvette system (4) can be rotated with steps of $0.01^{\circ}$. The reflected intensity is measured with a second photodiode (5). 
(Spectra Physics) of wavelength $632.8 \mathrm{~nm}$. With a beamsplitter and reference photodiode the laser intensity is measured and the signal is corrected for intensity fluctuations. A flow cell with BK 7 prism and PIN photodiode are placed on a rotation table, which is driven by a stepping motor making steps of $0.01^{\circ}$. The temperature of the liquid in the flow cell is stabilized with an NTC resistor and a Peltier element $\left(20.9 \pm 0.1{ }^{\circ} \mathrm{C}\right)$. Signal handling and stepping motor control are done with a personal computer. The silver layers of $53 \mathrm{~nm}$ were evaporated $(25 \AA / \mathrm{s}$ at $1 \times 10^{-6}$ mbar) on microscope glasses (Menzel Gläser), which are subsequently coupled to the prism with a matching oil with $n=1.518$ (Leitz). As a standard liquid (medium 4) a $0.1 \mathrm{M}$ phosphate buffer (PB) pH 7.15 in deionized water is used with $\epsilon=1.78$. For the media with higher dielectric constant we selected a liquid which mixes very well with water. Glycerol was chosen as it is unharmful for biomolecules/proteins. When the dielectric constant of the liquid becomes too high, the plasmon angle $\theta_{\mathrm{p}}$ is so large than it is no longer possible to reach surface plasmon resonance with the BK7 prism. Therefore we made solutions GL5 (0.1 M phosphate buffer $\mathrm{pH} 7.15$ in deionized water: glycerin $=5: 1 ; \epsilon=1.84$ ) and GL2.5 (0.1 $\mathrm{M}$ phosphate buffer pH 7.15 in deionized water: glycerin $=2.5: 1 ; \epsilon=1.88$ ).

The flow cell has a volume of $0.5 \mathrm{ml}$ and the buffer was changed by flowing $5-7 \mathrm{ml}$ of the new buffer through the cell. We checked this procedure by measuring the index of refraction of the bulk solution and the contents of the cell.

The procedure for characterizing a layer on top of the silver layer was as follows: After a first measurement of the SPR curve for a bare silver layer in PB and rinsing the cell with GL5, the SPR curve in GL5 was determined. Then the same was done with GL2.5. This cycle was repeated three times. Subsequently the whole procedure was followed for the silver layer with the additional dielectric layer.

The three measurements of a SPR curve under identical conditions should yield identical results. Initially this was not the case in our experiments, which was due to problems with the stability of the silver layer. We optimized the procedure by letting the bare silver layer stabilize for 1-2 days in PB before starting the experiment. This led to a stability of the minimum position of the SPR curve of about $0.002^{\circ} / \mathrm{hr}$.

We tested the method by determining $d_{3}$ and $\epsilon_{3}$ of a sputtered PTFE layer; for reference purposes this layer covered only part of the silver film, so that on one and the same slide the SPR curves of the bare silver film and the coated film could be compared. This can only be done when the thickness of the silver is constant over the whole layer area. With the optical system consisting of prism, matching oil and microscope slide the plasmon angle changed by $0.06-$ $0.1^{\circ} / \mathrm{mm}$ over the bare silver layer. We interpret this variation as being due to the curving of the slide and thickness variations of the oil layer. For a silver layer deposited directly on top of the prism the variation was $0-0.02^{\circ} / \mathrm{mm}$. Therefore we used silver layers evaporated on the prism for these PTFE experiments. In addition to this layer system, which we shall denote by configuration I, another layer system, configuration II, was prepared; here the silver film was partly covered with a single and partly with a double PTFE layer.

After this series of experiments intended to test the method, an adsorbed protein layer, consisting of the antibody anti Human Serum Albumine (aHSA) obtained from Sigma, was studied. First the measurements were performed by changing the buffer as described above for the bare silver layer. Then aHSA was adsorbed to the silver film from a $0.2 \mathrm{mg} / \mathrm{ml}$ aHSA solution in PB during one hour. After this adsorption the procedure for changing the buffer was repeated for the silver-protein configuration.

\section{Results}

\subsection{PTFE experiments}

Two experiments were performed with configuration I. By characterizing a layer we characterize it including its interface with the solution. In order to see whether the interface region of PTFE makes a sizeable contribution to the average layer characteristics, we also did two experiments with configuration II. Now the properties at the PTFE/solution interface are the same for both the single and the double PTFE layer. The stabilizing period of one day in PB 
takes care of the silver interface effects. During this period the drift curves of the silver layer with and without a PTFE layer are similar, so that also with PTFE layer the phosphate buffer reaches the silver interface. SPR curves were measured on both halves. From the SPR curve of the bare silver (in configuration I) we calculated the dielectric constant and thickness of the silver layer for the different bulk solutions with the method described in ref. [3]. For configuration II the same was done for the combination of the silver layer and the single PTFE layer.

The thickness of the additional PTFE layer which was subsequently characterized, was also measured with a surface profiler (Dektak 3030 ) by comparing the thickness of the single coated silver layer with that of the double coated silver layer. The same was done for the non and single coated silver layers. The re- sults of the SPR and surface profiler measurements are given in fig. 7 and table 1 . The dielectric constant of samples 3 and 4 could be determined more accurately as the value is close to the dielectric constants of the buffer solutions used. The results show

Table 1

Determination of the thickness and dielectric constant of the PTFE layers from fig. 7.

\begin{tabular}{lllll}
\hline Conf. & SPR & & & $\begin{array}{l}\text { Dektak } \\
d_{3} \pm 20(\AA)\end{array}$ \\
\cline { 2 - 5 } & & $d_{3}(\AA)$ & $\epsilon_{3}$ & \\
\hline I & sample 1 & $63 \pm 6$ & $2.14 \pm 0.03$ & 76 \\
I & sample 2 & $55 \pm 7$ & $2.06 \pm 0.03$ & 66 \\
II & sample 3 & $41 \pm 8$ & $1.829 \pm 0.004$ & 29 \\
II & sample 4 & $20 \pm 1$ & $1.829 \pm 0.004$ & 16 \\
\hline
\end{tabular}
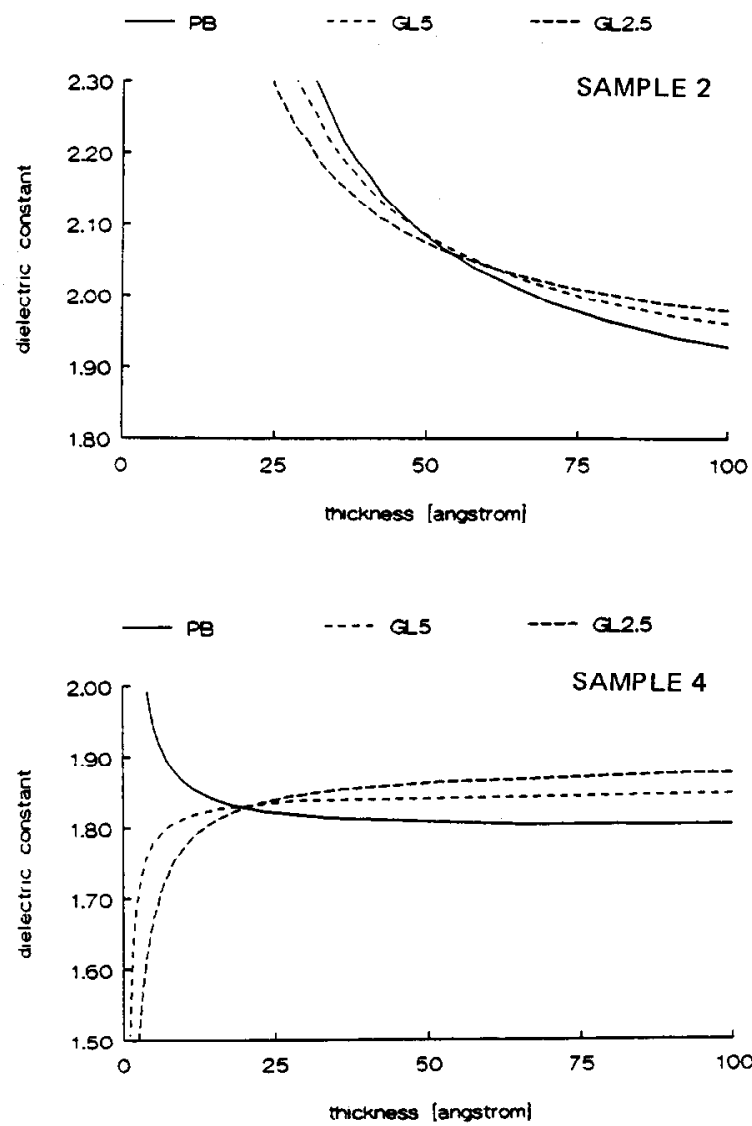

Fig. 7. The dielectric constant and thickness of four PTFE layers with configuration I (samples 1 and 2, top left and right) and configuration II (samples 3 and 4 , bottom left and right). 

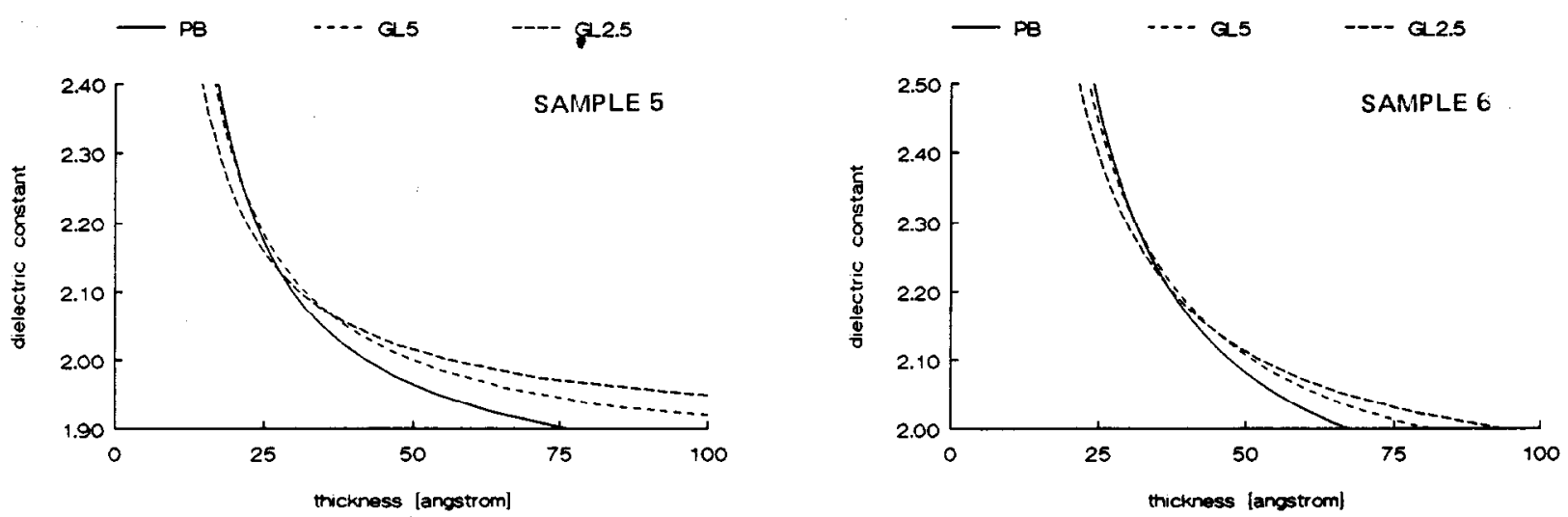

Fig. 8. The dielectric constant and thickness of two $\alpha$ HSA layers (samples 5 and 6).

that the dielectric constant of the top layer is different for configurations I and II and thus the nature of the interface can indeed contribute to the average dielectric constant. As it is beyond the scope of the present paper we will not discuss this point further.

\section{2. $\alpha H S A$}

We did two experiments with $\alpha \mathrm{HSA}$ on top of a silver layer. With eq. (3) the $\epsilon_{3}, d_{3}$ pairs were calculated and the results are given in fig. 8 and table 2. The thickness is small as only a small amount of aHSA was adsorbed to the silver. The dielectric constant is of the same order as the values given in ref. [5], but depends on the kind of metal used as a substrate.

\subsection{Accuracies}

The errors given in the above mentioned results were determined by averaging the three intersection points in figs. 7 and 8 . It is better to determine the accuracy by looking at the influence which the experimental errors have in the calculations.

\section{Table 2}

Determination of the thickness and dielectric constant of the two $\alpha$ HSA layers from figure 8.

\begin{tabular}{lll}
\hline & $d_{3}(\AA)$ & $\epsilon_{3}$ \\
\hline sample 5 & $29 \pm 7$ & $2.15 \pm 0.09$ \\
sample 6 & $38 \pm 6$ & $2.21 \pm 0.07$ \\
\hline
\end{tabular}

Both SPR curves of bare $53 \mathrm{~nm}$ silver and of protein coated $\left(d_{3}=27 \AA, \epsilon_{3}=2.14\right)$ silver were calculated in PB, GL5 and GL2.5. We varied the different parameters with the measuring errors and did the calculations with eq. (3). The results are given in table 3. This gives an error of about $6 \AA$ in $d_{3}$ and about 0.05 in $\epsilon_{3}$ for the aHSA layers.

The error is mainly determined by the error in $\Delta \theta_{\min }$. The errors compare well with the errors determined experimentally from the intersection points. The accuracy of the measurements can be improved when the stability is improved. Presently the limiting factor seems to be the stability of the silver layer. Therefore it may be important to find a protecting layer for the silver or to use a different metal layer. Another improvement can be made when the layer characteristics are approximately known beforehand, for example, after initial SPR experiments. From figs. 7 and 8 it can be seen that for very thin layers the error in $\epsilon_{3}$ increases, whereas for thicker layers the error in $d_{3}$ increases. To obtain the smallest errors in the layer characteristics, $\epsilon_{\mathrm{r}}$ must be

Table 3

The errors in the thickness and dielectric constant due to the experimental errors for a $27 \AA$ thick layer with $\epsilon=2.14$.

\begin{tabular}{llll}
\hline Variable & $\begin{array}{l}\text { Experimental } \\
\text { error }\end{array}$ & $\begin{array}{l}\text { Error } \\
\text { in } d_{3}(\AA)\end{array}$ & $\begin{array}{l}\text { Error } \\
\text { in } \epsilon_{\mathbf{3}}\end{array}$ \\
\hline$\epsilon_{\mathrm{r}}$ & 0.1 & 0.7 & 0.009 \\
$\epsilon_{4}$ & 0.001 & 0.3 & 0.003 \\
$\Delta \theta_{\min }$ & 0.02 & 5 & 0.036 \\
\hline
\end{tabular}


changed by changing wavelength or type of metal or by using adlayers, in order to obtain an optimum situation for the experiments. Also the dielectric constants of the bulk solutions could be chosen optimal, but this may mean that one has to use a different prism as well, in order to be able to excite the plasmon resonance.

\section{Conclusions}

We have presented a method for characterization of a thin dielectric layer on top of a silver layer by its thickness and dielectric constant. We use the SPR to do so and measure the shift in SPR parameters caused by the thin layer.

From the different possibilities to overcome the fact that an infinite number of $\epsilon_{3}, d_{3}$ combinations can cause the same shift in plasmon resonance curve, we prefer to change the dielectric constant of the surrounding bulk medium. We do realize that this may cause problems if thin dielectric layers are used, whose optical properties may be affected by the properties of the bulk medium. In view of this the results obtained are encouraging and we expect that the method described here will be of use if knowledge of the fundamental properties of layers at a surface are required.

An interesting point is that in experiments with PTFE layers we demonstrated that the dielectric constant of the PTFE layer is influenced by the interface with the surrounding solutions. This has to be taken into account in general when describing thin layers at the interface with a solvent.

\section{Appendix A}

With the approximations given in the theoretical part $r_{1234}$ can be written as

$$
\begin{aligned}
& r_{1234}=\frac{k_{x}-\left(\Gamma^{*}+\mathrm{i} \Gamma^{\mathrm{i}}\right)-\left(\Gamma^{+}-\mathrm{i} \Gamma^{\mathrm{rad}}\right)}{k_{x}-\left(\Gamma^{*}+\mathrm{i} \Gamma^{\mathrm{i}}\right)-\left(\Gamma^{+}+\mathrm{i} \Gamma^{\mathrm{rad}}\right)} r_{12}, \\
& R=\left|r_{1234}\right|^{2} \\
& =\left(1-\frac{4 \Gamma^{\mathrm{i}} \Gamma^{\mathrm{rad}}}{\left(k_{x}-\Gamma^{*}-\Gamma^{+}\right)^{2}+\left(\Gamma^{\mathrm{i}}+\Gamma^{\mathrm{rad}}\right)^{2}}\right)\left|r_{12}\right|^{2},
\end{aligned}
$$

with

$$
\begin{aligned}
& \Gamma^{*}=k_{x}^{0}+C / D, \quad \Gamma^{\mathrm{i}}=B / D, \\
& \Gamma^{+}=A \operatorname{Re}\left(r_{12}\right) \exp \left(2 \mathrm{i} k_{z 2} d_{2}\right) / D, \\
& \Gamma^{\mathrm{rad}}=A \operatorname{Im}\left(r_{12}\right) \exp \left(2 \mathrm{i} k_{z 2} d_{2}\right) / D, \\
& A=2 t_{4} t_{6}+\left(t_{1} t_{6}-t_{2} t_{6}+t_{4} t_{8}+t_{5} t_{8}\right) d_{3} k_{z 3}, \\
& B=t_{5} t_{8}-t_{1} t_{6}-\left(t_{5} t_{6}+t_{1} t_{8}\right) d_{3} k_{z 3}, \\
& C=\left(t_{1} t_{6}+t_{2} t_{6}+t_{4} t_{8}-t_{5} t_{8}\right) d_{3} k_{z 3}, \\
& D=t_{2} t_{9}-t_{6} t_{13}+\left(t_{3} t_{6}+t_{1} t_{7}\right. \\
& \left.\quad+t_{2} t_{7}+t_{5} t_{9}-t_{4} t_{9}-t_{8} t_{13}\right) d_{3} k_{z 3},
\end{aligned}
$$

with

$$
\begin{aligned}
& t_{1}=\epsilon_{3} \epsilon_{\mathrm{i}}\left(\epsilon_{\mathrm{r}}-\epsilon_{4}\right) \sqrt{\epsilon_{3} \epsilon_{\mathrm{r}}-\epsilon_{3} \epsilon_{4}-\epsilon_{\mathrm{r}} \epsilon_{4}}, \\
& t_{2}=2 \epsilon_{\mathrm{r}}^{2}\left(\epsilon_{3} \epsilon_{\mathrm{r}}-\epsilon_{3} \epsilon_{4}-\epsilon_{\mathrm{r}} \epsilon_{4}\right) \\
& t_{3}=2 \epsilon_{\mathrm{r}}^{2} \sqrt{\epsilon_{\mathrm{r}} \epsilon_{4}\left(\epsilon_{\mathrm{r}}-\epsilon_{4}\right)}, \\
& t_{4}=2 \epsilon_{\mathrm{r}}^{2} \epsilon_{3} \sqrt{\epsilon_{3} \epsilon_{\mathrm{r}}-\epsilon_{3} \epsilon_{4}-\epsilon_{\mathrm{r}} \epsilon_{4}}, \\
& t_{5}=2 \epsilon_{\mathrm{i}} \epsilon_{\mathrm{r}}\left(\epsilon_{\mathrm{r}} \epsilon_{3}-\epsilon_{3} \epsilon_{4}-\epsilon_{4} \epsilon_{\mathrm{r}}\right) \\
& t_{6}=\epsilon_{4}^{2}\left(\epsilon_{\mathrm{r}} \epsilon_{3}-\epsilon_{3} \epsilon_{4}-\epsilon_{4} \epsilon_{\mathrm{r}}\right) \\
& t_{7}=\epsilon_{4}^{2} \sqrt{\epsilon_{\mathrm{r}} \epsilon_{4}\left(\epsilon_{\mathrm{r}}-\epsilon_{4}\right)}, \\
& t_{8}=\epsilon_{3} \epsilon_{4}^{2} \sqrt{\epsilon_{3} \epsilon_{\mathrm{r}}-\epsilon_{3} \epsilon_{4}-\epsilon_{4} \epsilon_{\mathrm{r}}}, \\
& t_{9}=\epsilon_{3} \sqrt{\epsilon_{\mathrm{r}} \epsilon_{4}\left(\epsilon_{\mathrm{r}}-\epsilon_{4}\right)} \sqrt{\epsilon_{3} \epsilon_{\mathrm{r}}-\epsilon_{3} \epsilon_{4}-\epsilon_{4} \epsilon_{\mathrm{r}}}, \\
& t_{13}=2 \epsilon_{3} \epsilon_{\mathrm{r}} \sqrt{\left(\epsilon_{4}^{2} / \epsilon_{\mathrm{r}}\right)\left(\epsilon_{\mathrm{r}}-\epsilon_{4}\right)} \sqrt{\epsilon_{3} \epsilon_{\mathrm{r}}-\epsilon_{3} \epsilon_{4}-\epsilon_{4} \epsilon_{\mathrm{r}} .}
\end{aligned}
$$

From the equation for $R$ it can be seen that

$$
\begin{aligned}
k_{\min } & =I^{*}+\Gamma^{+} \\
& =k_{x}^{0}+C / D+A \operatorname{Re}\left(r_{12}\right) \exp \left(2 \mathrm{i} k_{z 2} d_{2}\right) / D \\
& =\frac{2 \pi}{\lambda} \sqrt{\frac{\epsilon_{\mathrm{r}} \epsilon_{4}}{\epsilon_{\mathrm{r}}-\epsilon_{4}}}+\frac{2 \pi}{\lambda} \frac{2}{\epsilon_{\mathrm{r}}+\epsilon_{4}}\left(\frac{\epsilon_{\mathrm{r}} \epsilon_{4}}{\epsilon_{\mathrm{r}}-\epsilon_{4}}\right)^{3 / 2} \\
& \times \operatorname{Re}\left(r_{12}\right) \exp \left(\frac{-4 \pi}{\lambda} d_{2} \frac{\epsilon_{\mathrm{r}}}{\sqrt{\epsilon_{\mathrm{r}}-\epsilon_{4}}}\right) \\
& +\left(\frac{2 \pi}{\lambda}\right)^{2} d_{3} \frac{\left(\epsilon_{\mathrm{r}} \epsilon_{4}\right)^{3 / 2}\left(\epsilon_{3} \epsilon_{\mathrm{r}}-\epsilon_{3} \epsilon_{4}-\epsilon_{4} \epsilon_{\mathrm{r}}+\epsilon_{3}^{2}\right)}{\epsilon_{3}\left(\epsilon_{\mathrm{r}}^{2}-\epsilon_{4}^{2}\right)\left(\epsilon_{\mathrm{r}}-\epsilon_{4}\right)} \\
& =k_{\min , \text { bare silver }} \\
& +\left(\frac{2 \pi}{\lambda}\right)^{2} d_{3} \frac{\left(\epsilon_{\mathrm{r}} \epsilon_{4}\right)^{3 / 2}\left(\epsilon_{3} \epsilon_{\mathrm{r}}-\epsilon_{3} \epsilon_{4}-\epsilon_{4} \epsilon_{\mathrm{r}}+\epsilon_{3}^{2}\right)}{\epsilon_{3}\left(\epsilon_{\mathrm{r}}^{2}-\epsilon_{4}^{2}\right)\left(\epsilon_{\mathrm{r}}-\epsilon_{4}\right)} .
\end{aligned}
$$


For $\epsilon_{4}=1$ this is the same equation as found by Kretschmann [2].

\section{References}

[1] M.T. Flanagan and R.H. Pantell, Electron. Lett. 20 (1984) 969.
[2] B. Liedberg, C. Nijlander and I. Lundström, Sens. Actuators 4 (1983) 299.

[3] H.E. de Bruijn, R.P.H. Kooyman and J. Greve, Appl. Optics 29 (1990) 1974.

[4] E. Kretschmann, Z. Phys. 241 (1971) 313.

[5] B.A. Iversson, P.O. Hegg, K.I. Lundström and U. Jönsson, Colloids Surf. 13 (1985) 169. 